Supporting Information for

\title{
A Point Mutation Converts Dihydroneopterin Aldolase to a Cofactor- Independent Oxygenase
}

Yi Wang, Gwynyth Scherperel, Kade D. Roberts, A. Daniel Jones, Gavin E. Reid, and Honggao

Yan

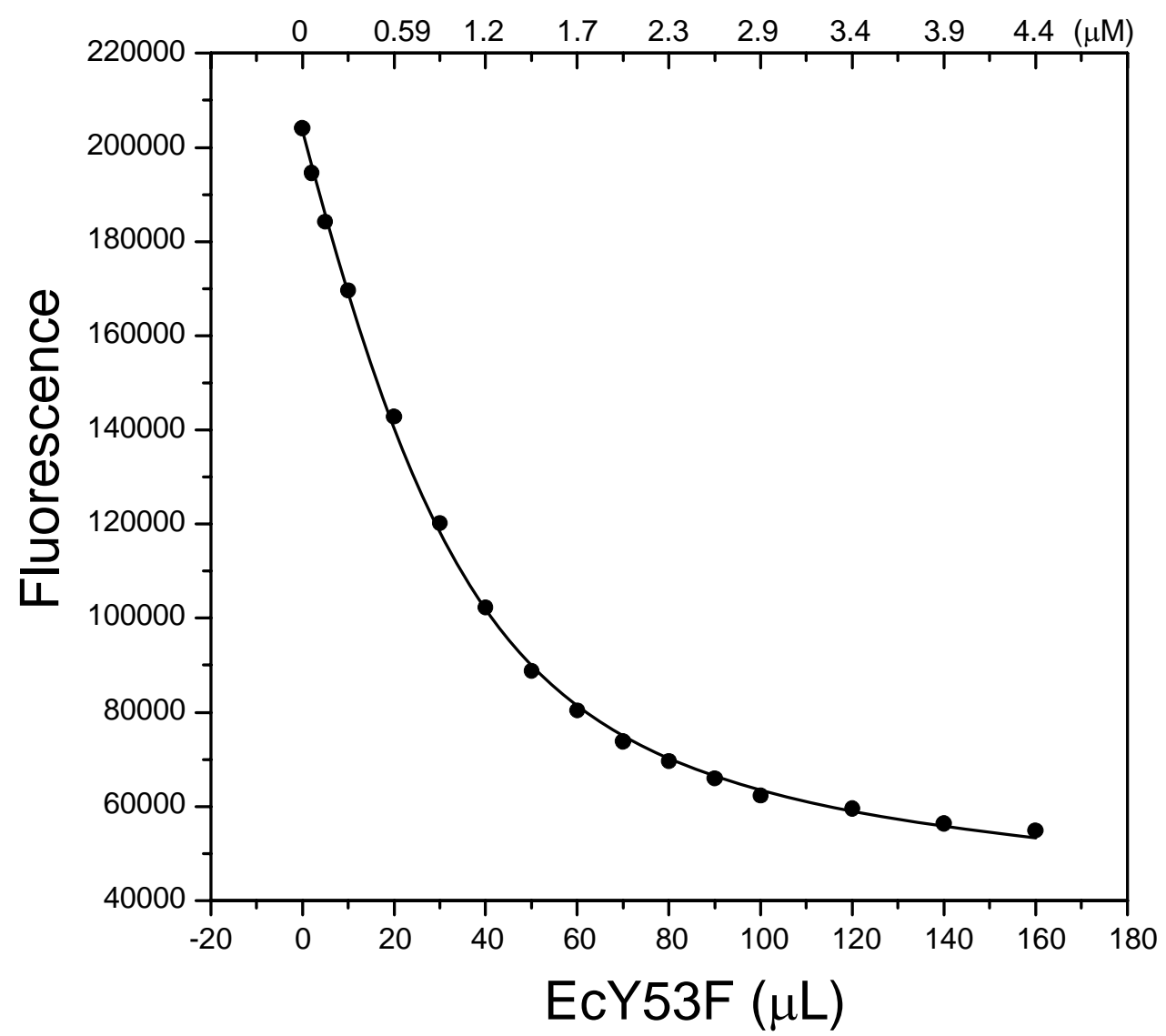

Figure S1. Binding of $\mathbf{1 0}$ to EcY53F at equilibrium. A $2 \mathrm{~mL}$ solution containing $1 \mu \mathrm{M} 10$ in 100 $\mathrm{mM}$ Tris-HCl, $\mathrm{pH} 8.3$ was titrated with EcY53F by adding aliquots of a $60 \mu \mathrm{M}$ EcY53F stock solution at $24^{\circ} \mathrm{C}$. The final concentration of $\mathbf{1 0}$ was $0.93 \mu \mathrm{M}$. The top axis indicates the EcY53F concentrations during the titration. A set of control data was obtained in the absence of $\mathbf{1 0}$ and was subtracted from the corresponding data set obtained in the presence of $\mathbf{1 0}$. The solid line was obtained by nonlinear least-squares regression as previously described. ${ }^{1}$ 

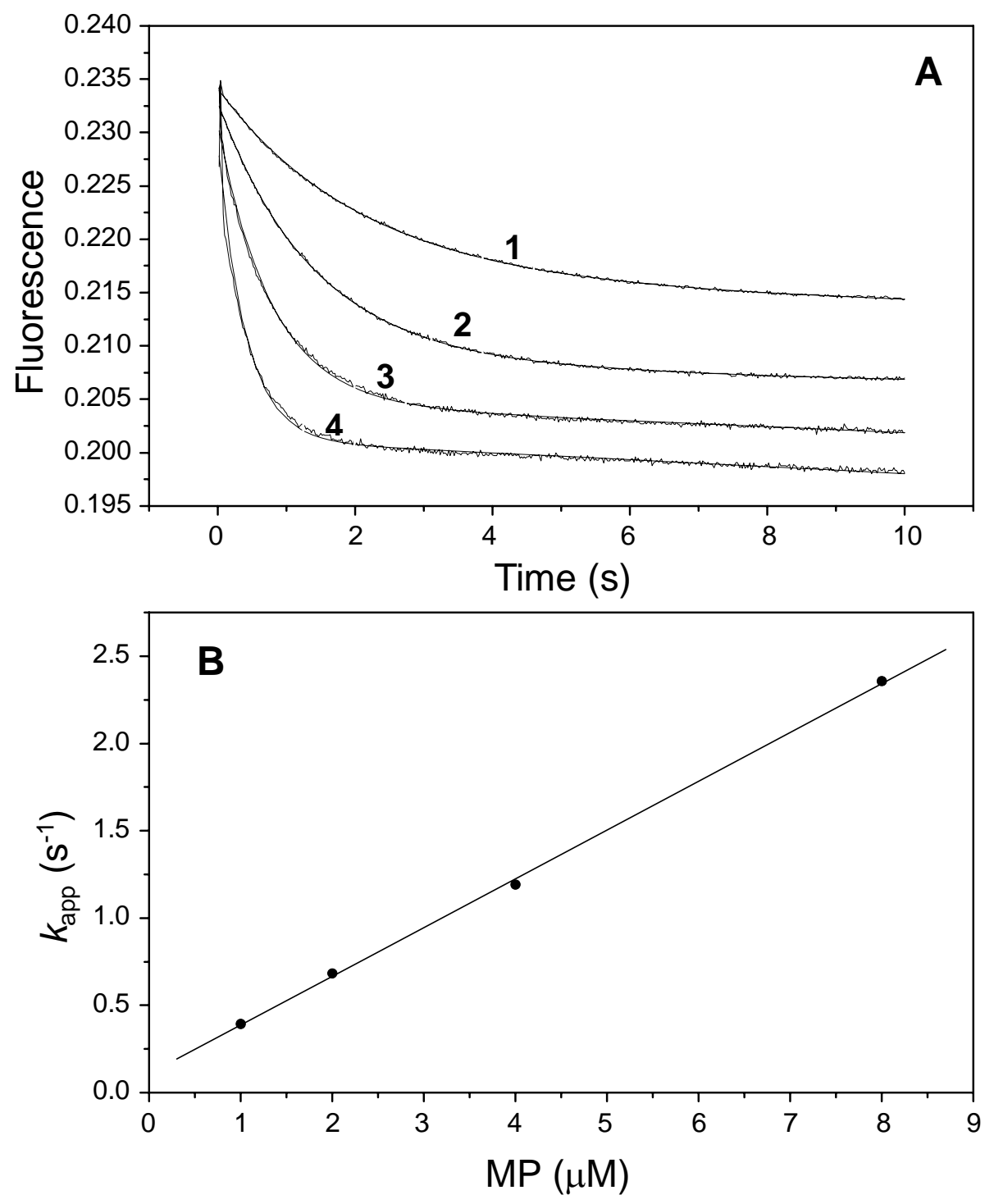

Figure S2. Stopped-flow analysis of the binding of 9 (MP) to EcY53F. The concentration of EcY53F was $0.2 \mu \mathrm{M}$, and the concentrations of $\mathbf{9}$ were 1, 2, 4 and $8 \mu \mathrm{M}$ for traces 1, 2, 3, and 4, respectively (A). All concentrations were those immediately after the mixing of the two syringe solutions. Both EcY53F and compound 9 were dissolved in $100 \mathrm{mM} 100 \mathrm{mM}$ Tris-HCl, pH 8.3. The fluorescent signals were rescaled so that they could be fitted into the figure with clarity. The solid lines were obtained by nonlinear regression as described in the Experimental Procedures section. B is a replot of the apparent rate constants vs. the concentrations $\mathbf{9}$. The solid line was obtained by linear regression. 

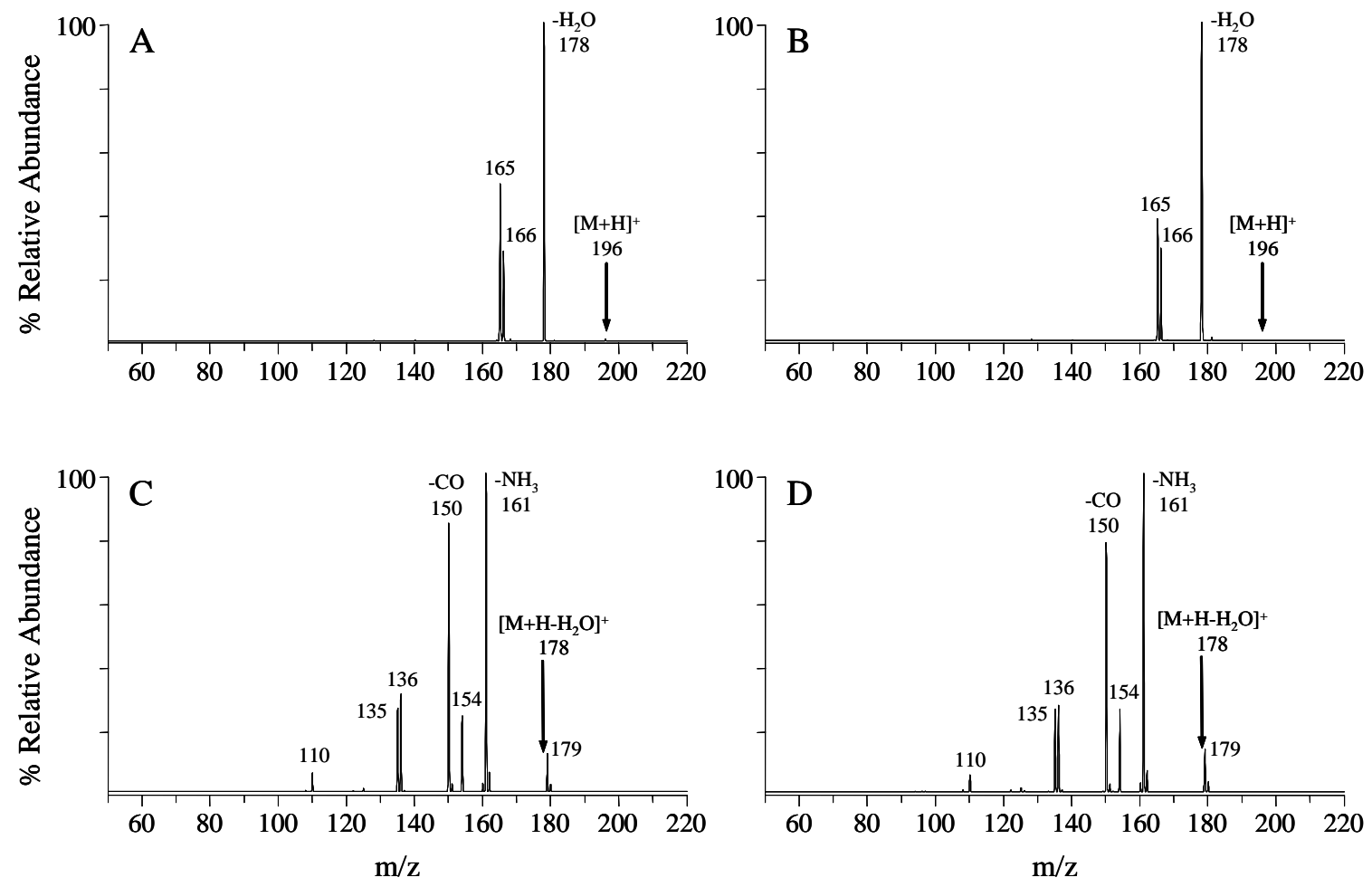

Figure S3. Multistage tandem mass spectrometry identification of the $\mathrm{m} / \mathrm{z} 196$ product from the reaction of 1 with SaDHNA. (A) CID MS/MS product ion spectrum of the ion at $\mathrm{m} / \mathrm{z} 196$ obtained from Figure 4A. (B) CID MS/MS product ion spectrum of the $\mathrm{m} / \mathrm{z} 196$ precursor ion obtained from a solution of commercially available 4. (C) CID $\mathrm{MS}^{3}$ product ion spectrum of the ion at m/z 178 in panel A. (D) CID MS ${ }^{3}$ product ion spectrum of the ion at m/z 178 in panel B. 

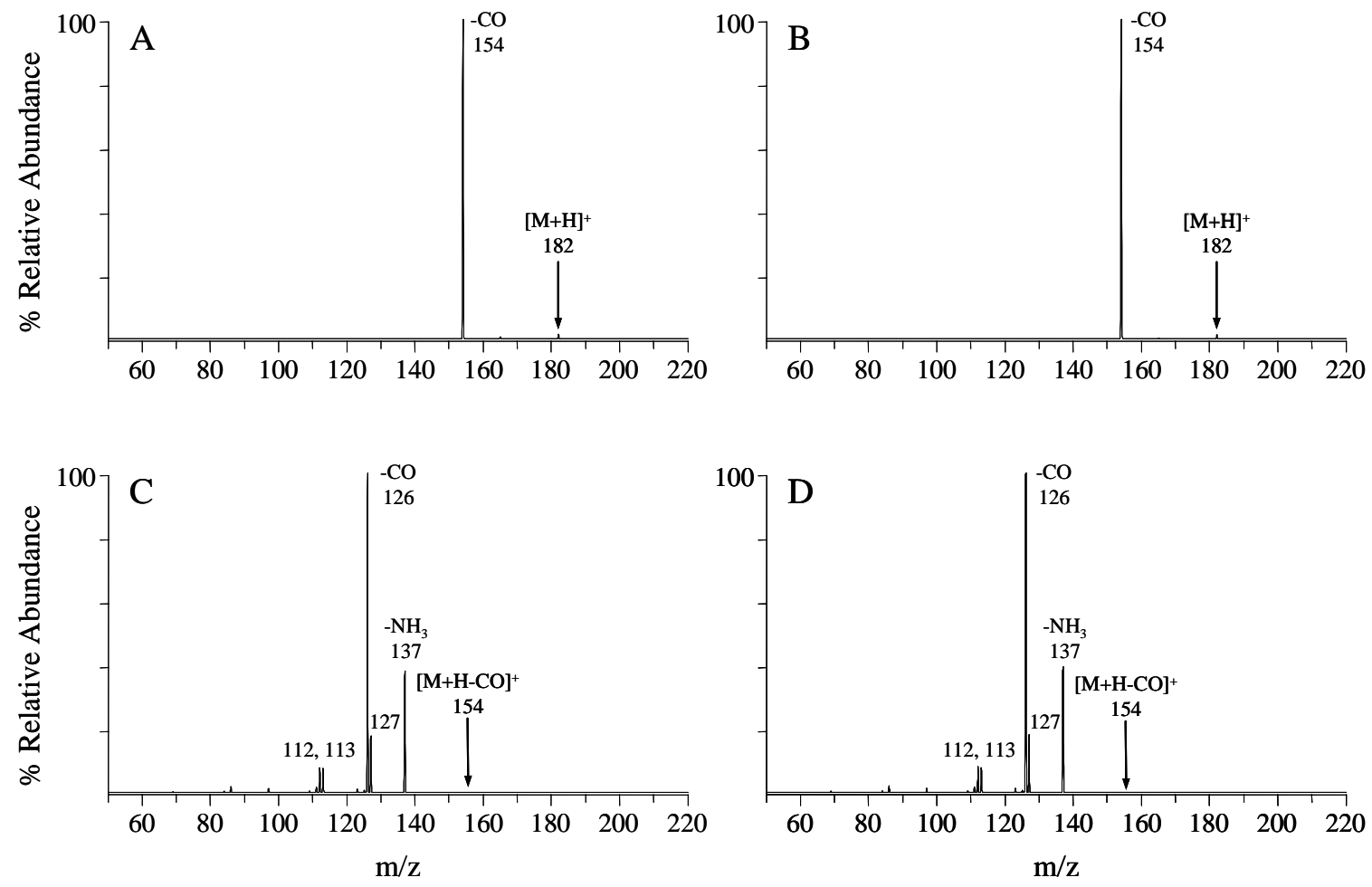

Figure S4. Multistage tandem mass spectrometry identification of the $\mathrm{m} / \mathrm{z} 182$ product from the reaction of 1 with SaY54F. (A) CID MS/MS product ion spectrum of the ion at $\mathrm{m} / \mathrm{z} 182$ obtained from Figure 4B. (B) CID MS/MS product ion spectrum of the $\mathrm{m} / \mathrm{z} 182$ precursor ion obtained from a solution of commercially available 6. (C) CID $\mathrm{MS}^{3}$ product ion spectrum of the ion at m/z 154 in panel A. (D) CID MS ${ }^{3}$ product ion spectrum of the ion at m/z 154 in panel B. 

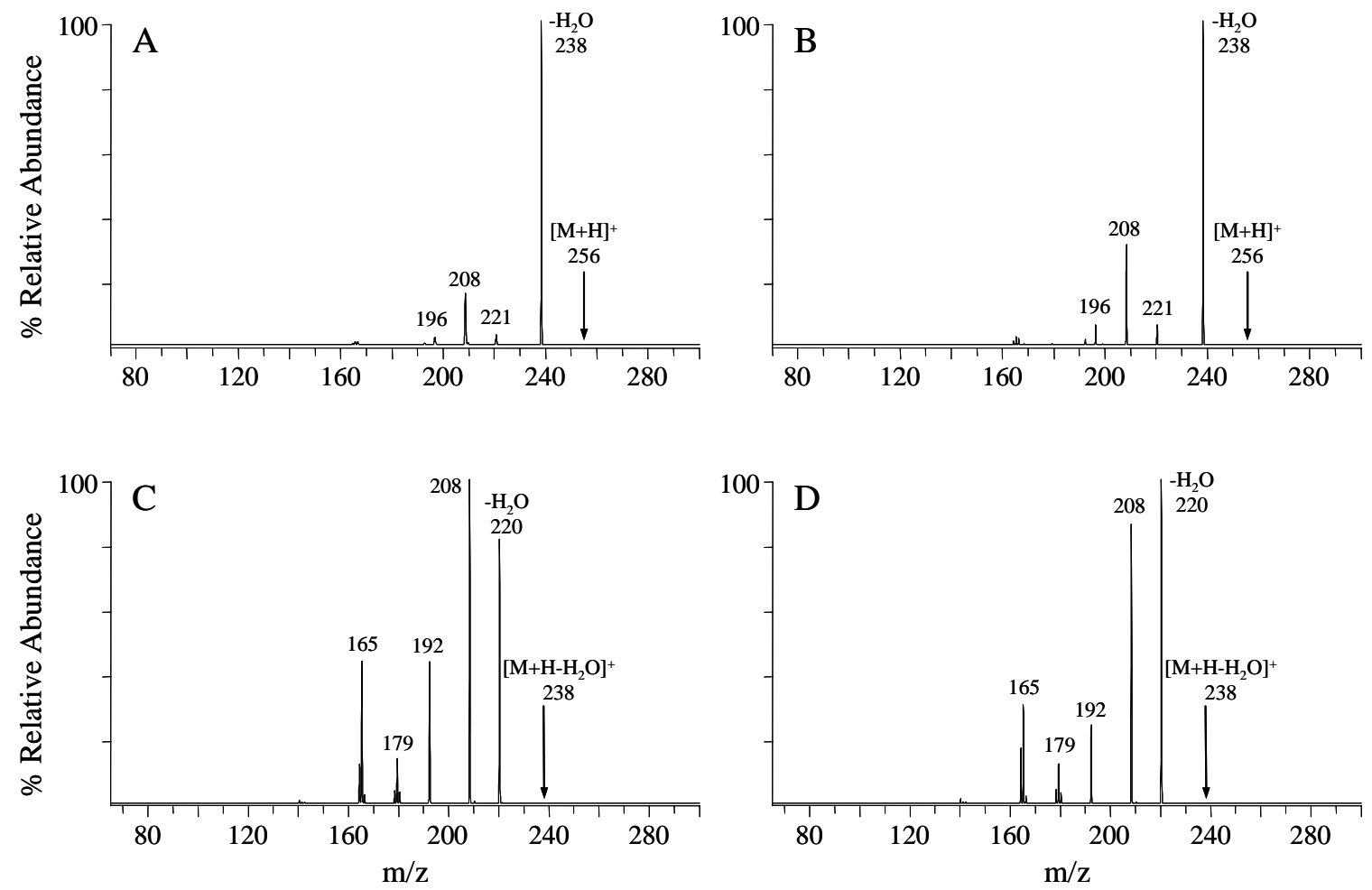

Figure S5. Multistage tandem mass spectrometry identification of the substrate 1. (A) CID MS/MS product ion spectrum of the ion at $\mathrm{m} / \mathrm{z} 256$ obtained from the reaction of $\mathbf{1}$ with SaDHNA at 0 min. (B) CID MS/MS product ion spectrum of the $\mathrm{m} / \mathrm{z} 256$ precursor ion obtained from a solution of commercially available 1. (C) CID MS ${ }^{3}$ product ion spectrum of the ion at $\mathrm{m} / \mathrm{z}$ 238 in panel A. (D) CID MS3 product ion spectrum of the ion at m/z 238 in panel B 

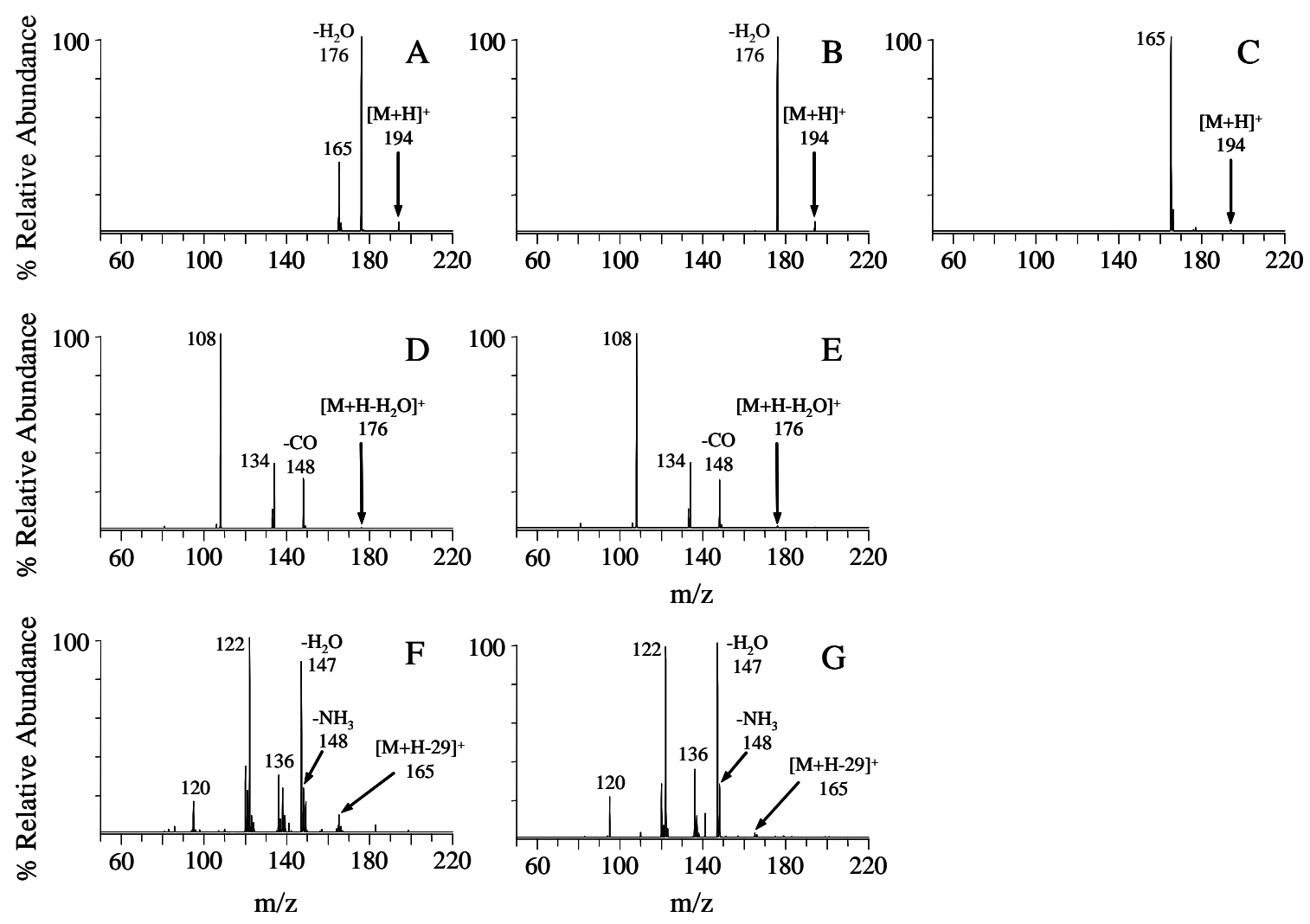

Figure S6. Multistage tandem mass spectrometry identification of the $\mathrm{m} / \mathrm{z} 194$ products from the reaction mixture of 1 catalyzed by SaY54F. (A) CID MS/MS product ion spectrum of the ion at m/z 194 obtained from the Figure 4B. (B) CID MS/MS product ion spectrum of the m/z 194 precursor ion obtained from a solution of commercially available 10. (C) CID MS/MS product ion spectrum of the m/z 194 precursor ion obtained from a solution of commercially available 7 . (D) CID $\mathrm{MS}^{3}$ product ion spectrum of the ion at $\mathrm{m} / \mathrm{z} 176$ in panel A. (E) CID MS ${ }^{3}$ product ion spectrum of the ion at $\mathrm{m} / \mathrm{z} 176$ in panel B. (F) CID $\mathrm{MS}^{3}$ product ion spectrum of the ion at $\mathrm{m} / \mathrm{z}$

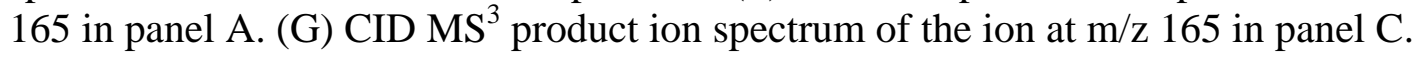



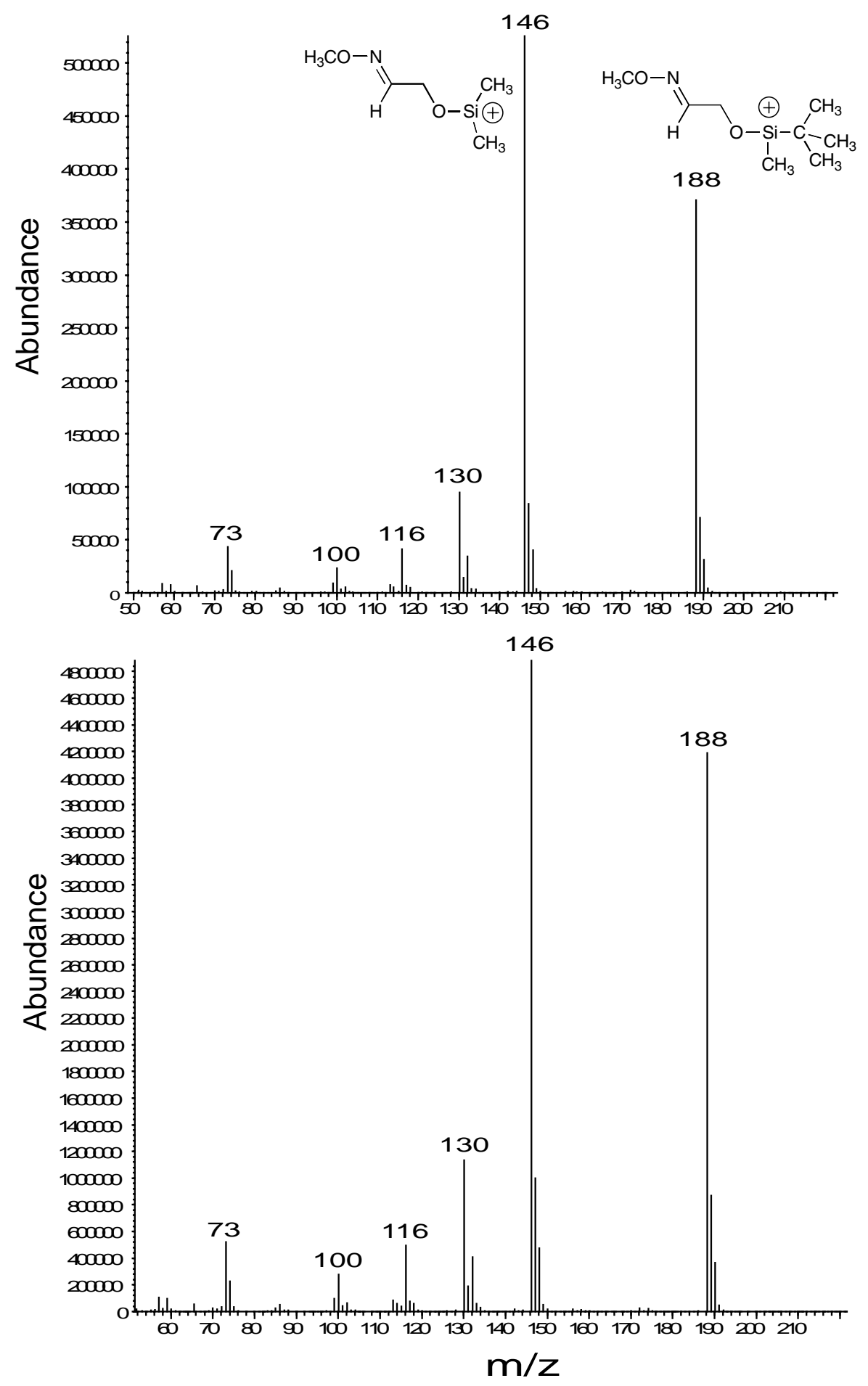

Figure S7. Electron ionization mass spectra of the tert-butyldimethylsilyl/methoxime derivatives of standard 3 (A) and the reaction mixture generated by SaY54F (B). The chromatographic retention times of the derivatives were $12.3 \mathrm{~min}$. 


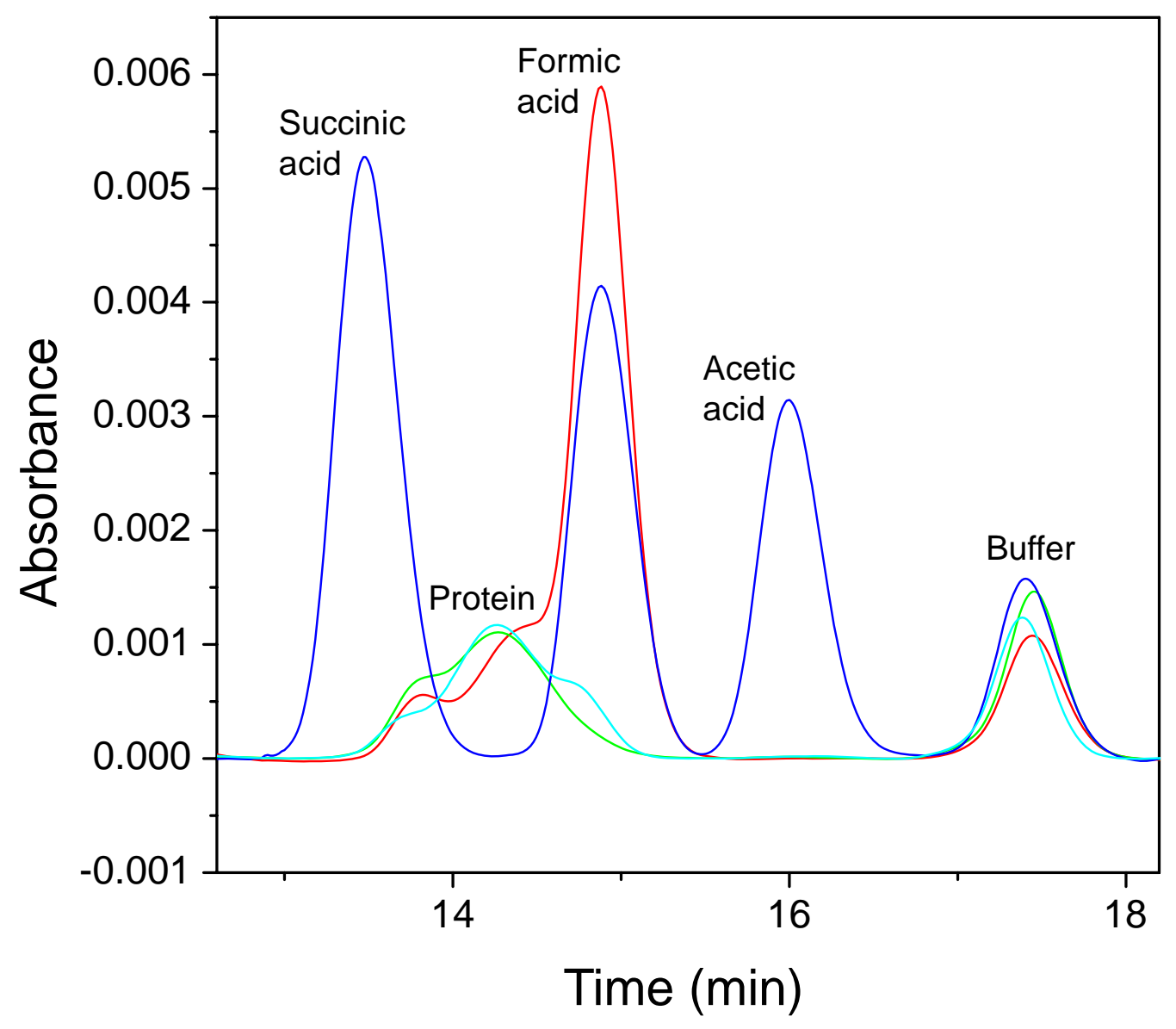

Figure S8. Identification of formic acid by HPLC. The red, cyan, green, and blue lines are the chromatogram of the SaY54F-catalyzed reaction mixture, the SaDHNA-catalyzed reaction mixture, the SaY54F protein solution, and a standard organic acid solution, respectively. The standard organic acid solution contained formic acid, acetic acid, and succinic acid

\section{Reference}

1. Li, Y.; Gong, Y.; Shi, G.; Blaszczyk, J.; Ji, X.; Yan, H., Biochemistry 2002, 41, 8777-8783. 